\title{
Remnants of embryonic arteries on the brain base: A case report*
}

\author{
Vasović Ljiljana\#, Jovanović Ivan, Ugrenović Slađana, Trandafilović Milena
}

Department of Anatomy, Faculty of Medicine, University of Niš, Niš, Serbia

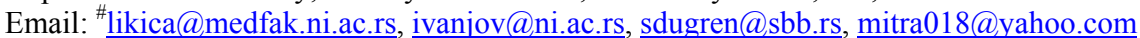

Received 1 November 2011; revised 30 January 2012; accepted 16 February 2012

\begin{abstract}
The posterior communicating artery (PCoA) arises from the cerebral part of the internal carotid artery and reaching the interpeduncular cistern unites with the posterior cerebral artery, marking the beginning of its post-communicating part. We accidentally found a case of an unusual origin and termination of the right PCoA associated with a presence of the right olfactory artery remnant in a 35-year-old male cadaver routinely dissected at Institute of Forensic Medicine. Presented vascular variations have not been reported previously in the literature. In the event of PCoA origin from the right middle cerebral artery normally developed from the cranial end of primitive internal carotid artery, as well as the PCoA junction with basilar tip, this PCoA could be the most cranial carotidbasilar anastomosis. Simultaneously, here presented right olfactory artery remnant could be the third variant of this persistent embryonic artery.
\end{abstract}

Keywords: Human Brain Base; Posterior Communicating Artery; Origin; Termination; Embryonic Arterial Remnants

\section{INTRODUCTION}

The early brain vesicles in the human embryo are supplied by paired primitive internal carotid arteries (ICAs). At about 4-mm stage, ICAs divide into cranial and caudal branches at the level of optic vesicles. The cranial branch will provide the anterior choroidal, middle cerebral and primitive olfactory arteries, as well as the anterior cerebral artery at its terminal end. The caudal branches give off the diencephalic, mesencephalic and posterior choroidal branches; they will later (5- to 6-mm stage) join with paired longitudinal neural plexus, which independently developed on the ventral side of the hindbrain.

\footnotetext{
"Conflict of interest statement: We declare that we have no conflict of interest.

${ }^{\#}$ Corresponding author.
}

Each of longitudinal neural plexus at this stage is supplied by some transitory vascular channels, i.e. cranially by the primitive trigeminal, caudally by the primitive proatlantal intersegmental, and laterally by the primitive otic and primitive hypoglossal arteries. Usually, paired longitudinal neural arterial plexus unite and form the basilar artery (BA) in $7-12 \mathrm{~mm}$ crown-rump length embryo. The caudal branch becomes the posterior cerebral artery (PCA), its proximal segment becoming later the posterior communicating artery (PCoA) [1].

It is fact that an intracerebral course and the areas supplied by PCoA branches are already well known [2], as well as its caliber and relationship with PCA [3-6].

The aim of this report was to show unusual origin and termination of the PCoA associated with other vascular variants.

\section{CASE REPORT}

An autopsy was performed in a 35-year-old man subsequent to his death in the Clinical centre of Niš because of the myocardial infarction. There was complete autopsy finding in the report of colleague at the Institute of Forensic Medicine in Niš (S-502/10); our examination was focused on the brain base arteries. External morphology (calibre, possible abnormalities) of these arteries was inspected with a magnifying glass and recorded on a film.

There was an angioarchitecture on the brain base without variations on the first look (Figure 1). However, we examined its right and left halves of the cerebral arterial circle $(\mathrm{CAC})$ and neighbouring arteries on the brain base.

Right half (Figure 2(a)): The cerebral part (C4) of ICA was visible; its outer diameter was $3.59 \mathrm{~mm}$. Its side branches were uncal (UA), anterior choroidal (AChA) and superior hypophyseal (SHA) arteries. The terminal ICA branches were anterior cerebral (ACA) and middle cerebral (MCA) arteries. The ACA was of normal caliber and course. The MCA trunk courved laterocaudally and about $12 \mathrm{~mm}$ after ICA bifurcation we had an impression that the PCoA was in continuation of the MCA. At the level of a "beginning" of the PCoA, the MCA trunk was ascend to the insula. An artery began ascending to the 
frontal lobe at the level of PCoA proximal third. It passed between the UA and AChA and in front of the proximal part of the AChA was divided into medial and lateral branches. The medial branch ascended in front of the $\mathrm{C} 4$ and pre-communicating part (A1) of the ACA to the olfactory tract; the lateral branch ascended to the olfactory tract passed in front of the UA and beginning part of the MCA. We marked this artery as a persistent primitive olfactory artery (POlA).

Vascular components of CAC left half were of normal calibers. In addition, there were three medial striate arteries or Heubner's arteries originated from $\mathrm{ACoA}$, lateral side of the A2 and sphenoid part (M1) of the MCA (Figure 2(b)).

The beginning of the PCoA could be presented using two annotations:

The first version. The PCoA had a common trunk with POlA and after its origin the PCoA continues as the single vessel (see Figure 2(a)).

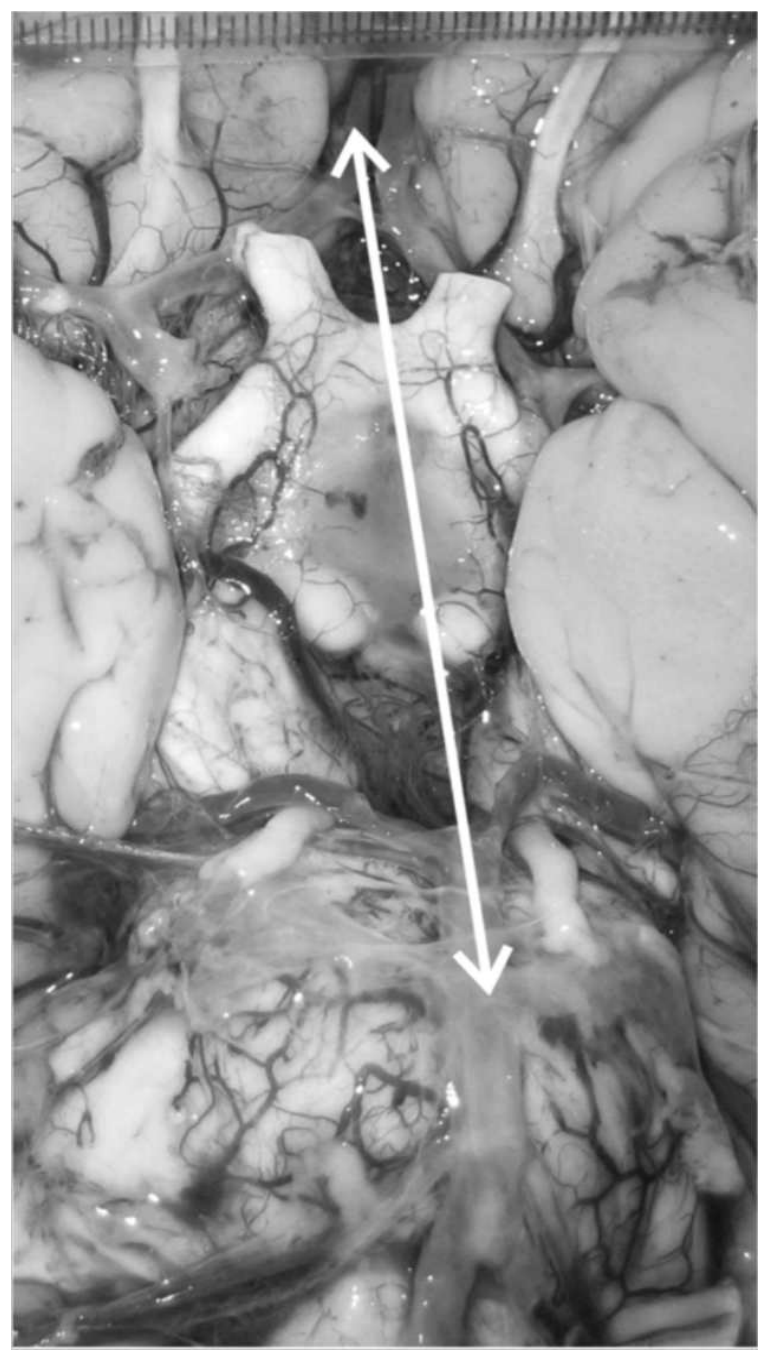

Figure 1. Some arteries of the carotid and vertebrobasilar systems of the brain base in a 35 -year-old male cadaver.

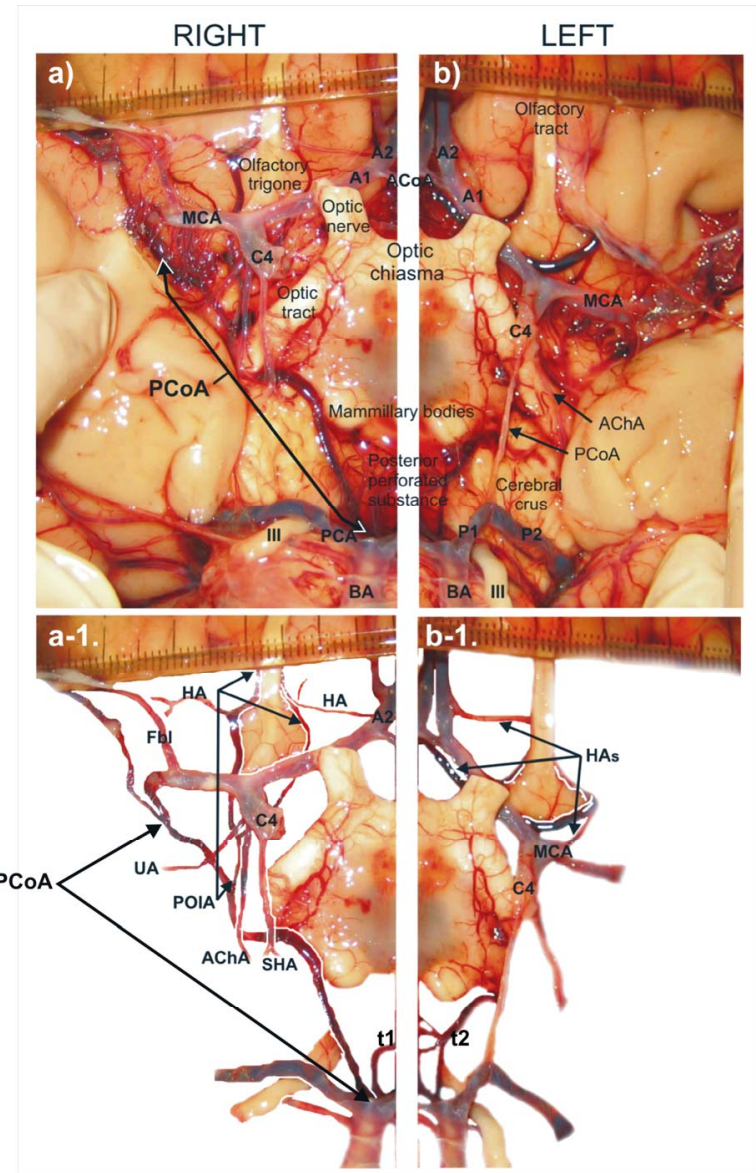

Right (a) and left (b) halves of the brain base arteries; C4 = Cerebral part of the internal carotid artery, $\mathrm{UA}=$ uncal artery, $\mathrm{AChA}=$ anterior choroidal artery, SHA = superior hypophyseal artery (it is dissected and because of that orientated to the right cerebral crus), $\mathrm{A} 1$ = pre-communicating part of the anterior cerebral artery, A2 = post-communicating part of the anterior cerebral artery, $\mathrm{ACoA}=$ anterior communicating artery, $\mathrm{HA}=$ Heubner artery, $\mathrm{MCA}=$ middle cerebral artery, $\mathrm{Fbl}=$ lateral frontobasal artery, $\mathrm{PCoA}$ $=$ posterior communicating artery, $\mathrm{POlA}=$ persistent primitive olfactory artery, $\mathrm{PCA}=$ posterior cerebral artery, $\mathrm{P} 1=$ pre-communicating part of the $\mathrm{PCA}, \mathrm{P} 2$ = post-communicating part of the PCA, BA = basilar artery, $\mathrm{t} 1-\mathrm{t} 2$ $=$ thalamoperforators, $\mathrm{III}=$ oculomotor nerve.

Figure 2. Unusual origin and termination of the right posterior communicating artery associated with persistent olfactory artery remnant.

The second version. The part of the MCA between its bend and lower margin of the POIA orificium could be persistent cranial end of the primitive ICA (MCA-PCoA anastomosis); the PCoA could be distally part of the vessel to the BA (Figures 3(a), (b)).

It is obvious that the "trunk" of the right PCoA in both images courved at the level of optic tract, cerebral crus and posterior perforated substance terminating at BA tip. The caliber of the right PCoA $(1.55 \mathrm{~mm})$ was twice larger than the left one $(0.70 \mathrm{~mm})$, but lower than caliber of the right PCA. There were side branches of the PCoA (posteromedial central, thalamotuberal and many hypothalamic branches, as well a branch to oculomotor nerve). The calibers of the C4, M1, A1 and P1 on both sides were 
approximately same values, respectively; BA caliber dominated in relation to the left or right C4 (Table 1). Summarizing, the CAC in this case has ten vascular components $(\mathrm{C} 4, \mathrm{~A} 1$, and PCoA on both sides, and unpaired ACoA, left P1, right $\mathrm{M} 1$ and right MCA-PCoA anastomosis or common right PCoA-POlA trunk).

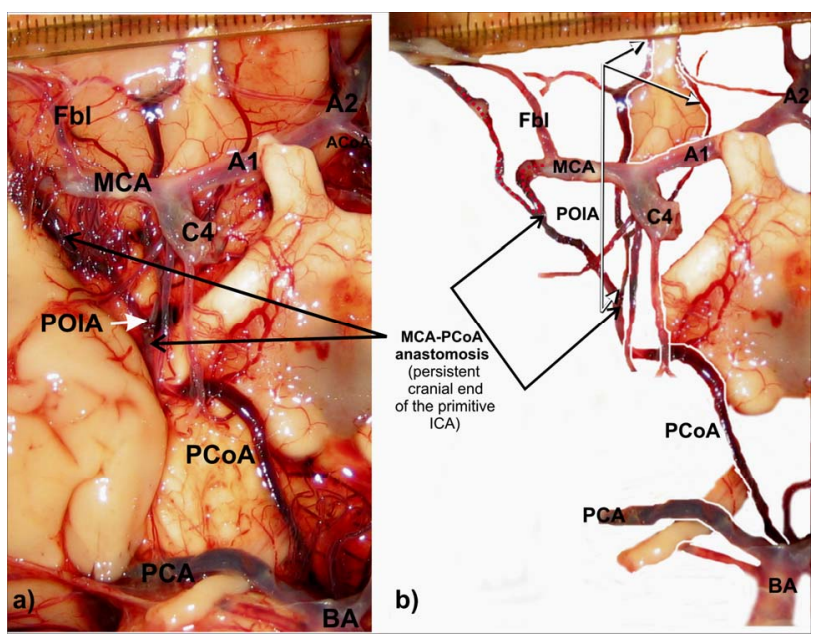

Right half of the brain base arteries (a-b): $\mathrm{C} 4=$ Cerebral part of the internal carotid artery, A1 = pre-communicating part of the anterior cerebral artery, $\mathrm{A} 2=$ post-communicating part of the anterior cerebral artery, $\mathrm{ACoA}=$ anterior communicating artery, $\mathrm{MCA}=$ middle cerebral artery, $\mathrm{Fbl}=$ lateral frontobasal artery, $\mathrm{PCoA}=$ posterior communicating artery, $\mathrm{POlA}=$ persistent primitive olfactory artery, $\mathrm{PCA}=$ posterior cerebral artery, $\mathrm{BA}=$ basilar artery.

Figure 3. Persistent cranial end of the right primitive internal carotid artery (MCA-PCoA anastomosis).

Table 1. Values of the outer diameter of selected brain base arteries.

\begin{tabular}{|c|c|c|c|}
\hline \multirow{2}{*}{ Brain base arteries } & \multicolumn{3}{|c|}{ Outer diameter $(\mathrm{mm})^{*}$} \\
\hline & Left & Single artery & Right \\
\hline $\begin{array}{l}\text { Internal carotid artery-cerebral part } \\
\text { (C4) }\end{array}$ & 3.10 & & 3.59 \\
\hline $\begin{array}{l}\text { Anterior cerebral artery- } \\
\text { pre-communicating part (A1) }\end{array}$ & 2.44 & & 2.48 \\
\hline Anterior communicating artery $(\mathrm{ACoA})$ & & 1.01 & \\
\hline Middle cerebral artery (MCA) & 2.94 & & 2.54 \\
\hline Posterior communicating artery (PCoA) & 0.70 & & 1.55 \\
\hline $\begin{array}{l}\text { Lateral branch of the olfactory artery } \\
\qquad(\mathrm{POlA})\end{array}$ & & & 1.30 \\
\hline Medial branch of the olfactory artery & & & 0.42 \\
\hline Uncal artery & & & 0.57 \\
\hline Anterior choroidal artery (AChA) & & & 0.73 \\
\hline Basilar artery (BA) & & 5.08 & \\
\hline Posterior cerebral artery (PCA) & & & 2.18 \\
\hline PCA—pre-communicating part (P1) & 2.30 & & \\
\hline
\end{tabular}

*Measurement was performed with ImageJ (http://rsb.info.nih.gov/ij/index. html).
A retrospective analysis including also 200 fetal brains [7] and 215 adult cadaver brain arteries obtained from 1984-1990 at the Department of Anatomy and from 2006 to 2011 at the Institute of Forensic Medicine in Niš did not discover similar PCoA variant associated with olfactory artery remnant.

\section{DISCUSSION}

We described associated vascular variations on the brain base in a 35-year old male cadaver. We found no case of the PCoA origin from the MCA in the literature, except the reports about different MCA duplication forms and/ or early bifurcations [8-10]. An origin of the right PCoA either in a continuation of ipsilateral MCA or in continuation of the persistent cranial end of ICA (MCAPCoA anastomosis) could be explained by some embryological facts quoted in Arey's book [11]. According to him, anomalous blood vessels may persist due to: 1) the choice of unusual paths in the primitive arterial plexuses, 2) the persistence of vessels normally obliterated or 3) incomplete development. Hypothetically, there were no cranial and caudal divisions of the primitive ICA, yet the caudal end was a continuation of the ICA cranial end, after origin of the POlA.

Carotid-vertebrobasilar anastomoses (primitive trigeminal, otic, hypoglossal and proatlantal intersegmental arteries) are embryonic presegmental arteries supplying the primitive vertebrobasilar system from the primitive ICA [12-15]. The origin and termination of the right PCoA indicates the most cranial of the embryonic vessels connecting the anterior and posterior cerebral circulations.

In the human fetus or adult, the PCoA arises either from the posterior, posterolateral or anterior aspect of the C4 part and reaching the interpeduncular cistern unites with the PCA, marking the beginning of its post-communicating part (P2) $[3,5]$. Normally, the PCoA distributes posteromedial central, thalamotuberal and mammillary arteries, chiasmatic and hypothalamic branches, artery of tuber cinereum and branch to oculomotor nerve [16], as in presented case. Here the right PCoA terminated at BA tip, as in a case of a 60-year-old man described by Varghese [17]. However, Varghese [17] also noted associated multiple vascular variations (presence of the left persistent trigeminal artery, absent ipsilateral PCoA, duplicated ipsilateral superior cerebellar artery, hypoplastic A1 part of contralateral ACA, azygous ACA and hypoplastic ipsilateral vertebral artery). Very interesting is a coincidence that in both cases the right PCoA was involved, as well as the persistent embryonic artery.

The question is why the right PCoA terminates at BA tip, while the left one divides ipsilateral PCA into P1 and P2 parts. Authors hypothesized that caudal end of the right primitive ICA is connected with the left longitudi- 
nal neural plexus, developing faster in relation to the right one, while the left caudal end of primitive ICA was fused with formed basilar trunk.

Basilar apex perforating branches were observed only in 7.3\% of fetal cases [18]. In this adult case two large thalamoperforators also originated from BA tip. Knowledge of the microvascular features of the thalamoperforating arteries is of an importance for neurosurgeons approaching vascular lesions of this area and also for neurologists and neurointerventionists for ischemic lesion involving BA and/or P1 parts [19]. In presented case, their finding was coincidental.

According to Padget [20], as quoted Komiyama [21], the cranial division of primitive ICA supplies the prosencephalon and constitutes the olfactory artery (POIA). This artery terminates in the nasal fossa, while the secondary artery constitutes the medial olfactory artery, which supplies the olfactory bulb. The medial olfactory artery becomes the ACA, while the terminal portion of the POlA usually regresses. Lateral olfactory branches of the POlA include the Heubner's artery, AChA, lateral striate artery, and later MCA. When the terminal portion of the POlA does not regress, it is called the persistent POlA. Nozaki [22] described two POlA variants. The POIA arising from ICA ran along the olfactory tract and made a hairpin bend to supply the distal ACA territory (variant 1). Kim and Lee [23] described also a case of the first variant. The POIA that rose from the ACA passing through the cribriform plate to supply the nasal cavity as the ethmoidal artery is the second variant. Here the right POlA remnant could be a new variant because of few reasons: 1) it begins from the persistent cranial end of primitive ICA or as a common trunk with PCoA; 2) its rostral part terminates around the olfactory trigone and tract, and 3) two right Heubner's arteries were formed originating from the post-communicating part (A2) of the ACA and from lateral branch of the POIA.

Development of the brain arteries from the cranial and caudal ends of the primitive ICA was of normal course on the left side.

\section{CONCLUSION}

In the event of PCoA beginning from the MCA originnated from the cranial end of primitive ICA, as well as the PCoA junction with basilar tip, presented right PCoA could be the most cranial carotid-basilar anastomosis. Simultaneously, here presented the right olfactory artery remnant could be the third variant of this persistent embryonic artery.

\section{ACKNOWLEDGEMENTS}

This work was supported by Ministry of Science and Technological Development of Republic of Serbia (contract grant numbers: 41018 and
175092). Authors thank Miodrag Zdravković, professor of Forensic medicine at Faculty of Medicine in Niš, for his patience and assistance during forensic dissection. Examination of brain arteries in adult cadavers was carried in accordance to the rules of the internal Ethics Committee (No. 01-9068-8).

\section{REFERENCES}

[1] Silver, J.M. and Wilkins, R.H. (1991) Persistent embryonic intracranial and extracranial vessels. In: Wilkins, R.H., Rengachary, S.S., Eds., Neurosurgery Update II: Vascular, Spinal, Pediatric, and Functional Neurosurgery. Health Professions Divisions, McGraw-Hill, New York, 90-98.

[2] Gabrovsky, N. (2002) Microanatomical bases for intraoperative division of the posterior communicating artery. Acta Neurochirurgica, 144, 1205-1211. doi:10.1007/s00701-002-1002-x

[3] Baskaya, M.K., Coscarella, E., Gomez, F. and Morcos, J.J. (2004) Surgical and angiographic anatomy of the posterior communicating and anterior choroidal arteries. Neuroanatomy, 3, 38-42.

[4] Vasović, L.P. (2004) The tenth vascular component in a rare form of the cerebral arterial circle of fetuses. Cells Tissues Organs, 178, 231-238. doi:10.1159/000083734

[5] Uz, A. and Mine, E.K. (2004) A morphological study of the posterior communicating artery. Folia Morphologica, 63, 397-399.

[6] Vasović, L.P., Jovanović, I.D., Ugrenović, S.Z. and Andjelković, Z.P. (2008) Normal subtypes of the posterior part of the cerebral arterial circle in human fetuses. Surgical Neurology, 70, 287-294. doi:10.1016/j.surneu.2007.06.087

[7] Vasović, L. (1990) Morphological characteristics of the cerebral arterial circle with different origin of the vertebral arteries (in Serbian). Thesis of the Faculty of Medicine, Niš.

[8] Teal, J.S., Rumbaugh, C.L., Bergeron, R.T. and Segall, H.D. (1973) Anomalies of the middle cerebral arteries: accessory artery, duplication, and early bifurcation. American Journal of Roentgenology, 118, 567-575.

[9] Uchino, A., Kato, A., Takase, Y. and Kudo, S. (2000) Middle cerebral artery variations detected by magnetic resonance angiography. European Radiology, 10, 560563. doi: $10.1007 / \mathrm{s} 003300050960$

[10] Dimmick, S.J. and Faulder, K.C. (2009) Normal variants of the cerebral circulation at multidetector CT angiography. RadioGraphics, 29, 1027-1043. doi:10.1148/rg.294085730

[11] Arey, B.L (1954) Developmental anatomy. Sixth Edition. Saunders, Philadelphia, 364-373.

[12] Caldemeyer, K.S., Carrico, J.B. and Mathews, V.P. (1998) The radiology and embryology of anomalous arteries of the head and neck. American Journal of Roentgenology, 170, 197-203.

[13] Vasović, L., Milenković, Z., Jovanović, I., Cukuranović, R., Jovanović, P. and Stefanović, I. (2008) Hypoglossal 
artery: A review of normal and pathological features. Neurosurgical Review, 31, 385-395. doi:10.1007/s10143-008-0145-5

[14] Vasović, L., Mojsilović, M., Andelković, Z., Jovanović. I., Arsić, S., Vlajković, S. and Milenković, Z. (2009) Proatlantal intersegmental artery: A review of normal and pathological features. Child's Nervous System, 25, 411421. doi:10.1007/s00381-008-0765-7

[15] Vasović, L., Arsić, S., Vlajković, S., Jovanović, I., Jovanović, P., Ugrenović, S. and Anđelković, Z. (2010) Otic artery: A review of normal and pathological features. Medical Science Monitor, 16, RA101-109.

[16] Dauber, W. (2007) Posterior communicating artery. In: Dauber, W., Ed., Pocket Atlas of Human Anatomy, 5th Revised Edition, Thieme, Stuttgart, New York, 246.

[17] Varghese, S.P.J. (2007) Persistent trigeminal artery and associated vascular variations. Australasian Radiology, 51, B31-B33. doi:10.1111/j.1440-1673.2007.01828.x

[18] Vasović, L., Jovanović, I., Ugrenović, S., Stojanov, D. and Radovanović, Z. (2008) Basilar bifurcation: A comparison of prenatal and postnatal cases. Neuroana- tomy, 7, 66-71.

[19] Park, S.Q., Bae, H-G., Yoon, S-M., Shim J-J., Yun, I-G. and Choi, S-K. (2010) Morphological characteristics of the thalamoperforating arteries. Journal of Korean Neurosurgical Society, 47, 36-41. doi: $10.3340 / \mathrm{jkns} .2010 .47 .1 .36$

[20] Padget, D.H. (1948) The development of the cranial arteries in the human embryo. Contrbutions to Embryology, 32, 205-261.

[21] Komiyama, M. (2012) Persistent primitive olfactory artery. Surgical and Radiologic Anatomy, 34, 97-98. http://web.mac.com/masakomiyama/Kodomo/\%E8\%AB $\% 96 \%$ E6\%96\%87 files/PDF127.pdf

[22] Nozaki, K., Taki, W., Kawakami, O. and Hashimoto, N. (1998) Cerebral aneurysm associated with persistent primitive olfactory artery aneurysm. Acta Neurochirurgica, 140, 397-402. doi:10.1007/s007010050114

[23] Kim, M.S. and Lee, G.J. (2011) Diagnosis of persistent primitive olfactory artery using computed tomography angiography. Journal of Korean Neurosurgical Society, 49, 290-291. doi:10.3340/jkns.2011.49.5.290 